\title{
Alpha-fetoprotein-L3: Useful or Useless for Hepatocellular Carcinoma?
}

Editor Liver Cancer

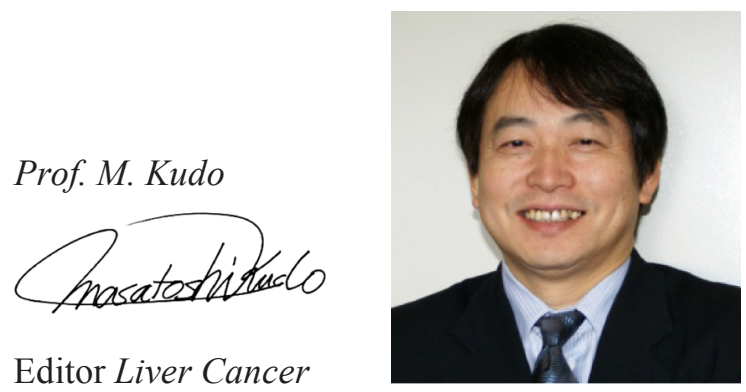

Japan is the only country where alpha-fetoprotein (AFP)-L3 can be measured in the routine clinical setting. Since AFP-L3 examination has been covered by health insurance in Japan for over 10 years, it is now routinely measured during general medical checkups for patients with chronic liver disease. To increase the specificity of AFP, the AFP-L3 glycoform can be used as a measure of cancerous changes in the AFP composite carbohydrate moiety. The most frequently used cut-off value is $10 \%$ [1], which gives a positive rate of $0 / 71(0.0 \%)$, $1 / 90$ (1.1\%), 0/13 (0.0\%), 0/14 (0.0\%), and 18/82 (22.0\%) for chronic hepatitis, hepatic cirrhosis, dysplastic nodules, early hepatocellular carcinoma, and advanced hepatocellular carcinoma, respectively. This yields a sensitivity of $18.8 \%$ and a specificity of $99.4 \%$. Therefore, although AFP-L3 has high specificity, due to its low sensitivity, it is considered to be of limited use in screening. It is possible, however, to diagnose a marginally higher number of patients with advanced hepatocellular carcinoma [2]. Furthermore, as AFP-L3 may be elevated during hepatic failure, interpretation should be made with caution.

AFP-L3 dynamics are largely related to the degree of biological malignancy of hepatocellular carcinoma $[2,3]$. Results of pathological investigations conducted on patients who have undergone hepatic resection showed that those with infiltrative growth, capsular invasion, septum formation, portal vein infiltration, and hepatic vein infiltration were significantly more likely to have AFP-L3-positive (>10\%) cancer [4].

To investigate cases of hepatocellular carcinoma for which therapeutic intervention was given and in which tumor markers were measured chronologically, an investigation was conducted prior to therapeutic intervention with a group of 196 AFP-L3-positive (>10\%) patients and a group of 645 AFP-L3-negative patients. When the overall survival rate was compared between the AFP-L3-positive and negative groups, the latter group showed more favorable survival than the former group $(\mathrm{p}<0.001)$. When the AFP-L3 dynamics before and at six months after treatment were compared, 599 cases remained negative, 113 remained positive, 83 underwent conversion from positive to negative (negative conversion), and 46 underwent conversion from negative to positive (positive conversion). When survival rates were investigated, the constantly negative and negative conversion groups had more favorable survival rates than the constantly positive and positive conversion group. In other words, even though there are AFP-L3-positive cancers with poor prognosis and a 
high degree of biological malignancy, when negative conversion is induced from therapeutic intervention, a survival rate equivalent to that of AFP-L3-negative cancer can be obtained.

Recently, highly sensitive AFP-L3 measurements have become available, and the usefulness of these has increased rapidly [5, 6]. Using conventional methods, AFP-L3 measurement was only possible at AFP levels $>10 \mathrm{ng} / \mathrm{ml}$, whereas using the highly sensitive method enables measurement at AFP levels $>2 \mathrm{ng} / \mathrm{ml}$. The highly sensitive AFP-L3 measurements are particularly effective in patients with low AFP values. In an investigation of 270 hepatocellular carcinoma patients with an AFP value $>20 \mathrm{ng} / \mathrm{ml}$ and a Child-Pugh score of A or B [5], when the cut-off value was set at 5\%, the conventional AFP-L3 measurement method yielded a sensitivity of $7 \%$ and a specificity of $98.5 \%$. Conversely, the highly sensitive method yielded a sensitivity of $41.5 \%$ and a specificity of $85.1 \%$, indicating that the sensitivity of the highly sensitive method was significantly higher than that of the conventional method $(\mathrm{p}<0.05)$.

Furthermore, the sensitivity for Stage I $(n=89)$ and Stage II $(n=127)$ was $4.5 \%$ and $2.4 \%$, respectively when the cut-off value using the conventional method was set at $10 \%$, while the sensitivity for the same stages was more favorable at $34.8 \%$ and $42.5 \%$, respectively when the cut-off value using the highly sensitive method was set at $5 \%$. In terms of prognostic assessment, when using the conventional method with the cut-off value set at $10 \%$, no significant difference was observed between those with values $>10 \%$ or $<10 \%$. However, reports indicate that when using the highly sensitive method with a cut-off value of $5 \%$, patients with values $<5 \%$ have a significantly more favorable prognosis than those with values $>5 \%(\mathrm{p}<0.01)$. In addition, even in patients with an AFP $<10 \mathrm{ng} / \mathrm{ml}$, when the cut-off value is set at $5 \%$ using the highly sensitive method, patients with values below $5 \%$ have a significantly more favorable prognosis than those with values $5 \%$ or above $(p=0.035)$. With regard to prognosis and the use of AFP-L3, multivariate analysis using the Cox proportional hazard model showed the following. For values of $5 \%$ and above the hazard ratio was 1.697 and $95 \%$ confidence interval was $1.066-3.440, p=0.026$. For treatment consisting of nonhepatic resection the hazard ratio was 3.627 and $95 \%$ confidence interval was $2.066-6.708$, $\mathrm{p}<0.001$. These findings enabled factors contributing to prognosis to be selected [5].

In conclusion, AFP-L3, in particular its high sensitivity measurement, is extremely useful as an index of prognostication and for the degree of biological malignancy of hepatocellular carcinoma. Consequently, it is highly expected that AFP-L3 will become more popular worldwide, and not just in Japan.

\section{References}

1 Shimizu K, Katoh H, Yamashita F, Tanaka M, Tanikawa K, Taketa K, et al: Comparison of carbohydrate structures of serum alpha-fetoprotein by sequential glycosidase digestion and lectin affinity electrophoresis. Clin Chim Acta 1996;254:23-40.

-2 Kumada T, Nakano S, Takeda I, Kiriyama S, Sone Y, Hayashi K, et al: Clinical utility of Lens culinaris agglutinin-reactive alpha-fetoprotein in small hepatocellular carcinoma: special reference to imaging diagnosis. J Hepatol 1999;30:125-130.

-3 Tamura Y, Igarashi M, Suda T, Wakai T, Shirai Y, Umemura T, et al: Fucosylated fraction of alpha-fetoprotein as a predictor of prognosis in patients with hepatocellular carcinoma after curative treatment. Dig Dis Sci 2010;55:2095-2101.

-4 Tada T, Kumada T, Toyoda H, Kiriyama S, Sone Y, Tanikawa M, et al: Relationship between Lens culinaris agglutinin-reactive alpha-fetoprotein and pathologic features of hepatocellular carcinoma. Liver Int 2005;25:848-853.

5 Toyoda H, Kumada T, Tada T, Kaneoka Y, Maeda A, Kanke F, et al: Clinical utility of highly sensitive Lens culinaris agglutinin-reactive alpha-fetoprotein in hepatocellular carcinoma patients with alpha-fetoprotein $<20$ ng/ml. Cancer Sci 2011;102:1025-1031.

6 Tamura Y, Igarashi M, Kawai H, Suda T, Satomura S, Aoyagi Y: Clinical advantage of highly sensitive onchip immunoassay for fucosylated fraction of alpha-fetoprotein in patients with hepatocellular carcinoma. Dig Dis Sci 2010;55:3576-3583. 